\title{
Decreasing of the $L^{1}$ norm and mass conservation for Porous Medium Equations with advection
}

\section{Decrescimento da norma $L^{1}$ e conservação de massa para equações de meios porosos com advecção}

Instituto Federal de Educação, Ciência e Tecnologia do Rio Grande do Sul (IFRS), Campus Canoas

Canoas, RS, Brasil

nicolau.diehl@canoas.ifrs.edu.br

Lucinéia Fabris

Universidade Federal de Santa Maria (UFSM), Campus Cachoeira do Sul Cachoeira do Sul, RS, Brasil lucineia.fabris@ufsm.br

\section{Informações do Artigo}

(c) (7) \&)

\section{Histórico do Artigo}

Submissão: 04 de maio de 2018.

Aceite: 21 de novembro de 2018.

\section{Keywords}

Mass Conservation

Decreasing of the $L^{1}$ Norm

Porous Medium Equations

\section{Abstract}

In this paper, we show that the $L^{1}$ norm of the bounded weak solutions of the Cauchy problem for general degenerate parabolic equations of the form

$$
u_{t}+\operatorname{div} f(x, t, u)=\operatorname{div}\left(|u|^{\alpha} \nabla u\right), \quad x \in \mathbb{R}^{n}, t>0,
$$

where $\alpha>0$ is constant, decrease, under fairly broad conditions in advection flow $f$. In addition, we derive the mass conservation property for positive (or negative) solutions.

\section{Palavras-chave}

Conservação de Massa

Decrescimento da Norma $L^{1}$

Equações de Meios Porosos

\section{Resumo}

Neste artigo, mostramos que a norma $L^{1}$ de soluções fracas limitadas do problema de Cauchy para equações parabólicas degeneradas gerais, da forma

$$
u_{t}+\operatorname{div} f(x, t, u)=\operatorname{div}\left(|u|^{\alpha} \nabla u\right), \quad x \in \mathbb{R}^{n}, t>0 \text {, }
$$

onde $\alpha>0$ é constante, decresce, sob condições bastante amplas para o fluxo de advecção $f$. Além disso, derivamos a propriedade de conservação de massa para soluções positivas (ou negativas).

\section{Introduction}

The Porous Medium Equations have been studied for a long time and appear as models for several areas, such as plasma physics to filtration in porous media, thin films, Riemannian geometry, among others. In this paper, our main goal is to obtain the decreasing of the $L^{1}$ norm for bounded 
(signed) weak solutions of the Cauchy problem

$$
\begin{aligned}
u_{t}+\operatorname{div} f(x, t, u) & =\operatorname{div}\left(|u|^{\alpha} \nabla u\right) \quad x \in \mathbb{R}^{n}, t>0, \\
u(\cdot, 0) & =u_{0} \in L^{1}\left(\mathbb{R}^{n}\right) \cap L^{\infty}\left(\mathbb{R}^{n}\right),
\end{aligned}
$$

given constants $\alpha>0$ and $f \in C^{1}$ satisfying

(f1) Let be $M>0$, and $T<T_{*}$. Then, for all $(x, t, v) \in \mathbb{R}^{n} \times[0, T] \times[-M, M]$, exists $K=K(M, T)>0$ such that $|f(x, t, v)|<K|v|$.

Besides that, we will derive the mass conservation property for positive (or negative) solutions.

In the case of $f$ not depending on $x$ and $t$, we have, in particular, the problem

$$
\begin{aligned}
u_{t}+\operatorname{div} f(u) & =\operatorname{div}\left(|u|^{\alpha} \nabla u\right) \quad x \in \mathbb{R}^{n}, t>0, \\
u(\cdot, 0) & =u_{0} \in L^{1}\left(\mathbb{R}^{n}\right) \cap L^{\infty}\left(\mathbb{R}^{n}\right),
\end{aligned}
$$

whose solutions exhibit a lot of known properties of parabolic problems in a conservative way as, for example, regularity, decay in $L^{1}$ norm, mass conservation and comparison properties. However, in the general case (i.e., $f=f(x, t, u)$ ) some of these properties are no longer valid in general, such as decay in $L^{q}$ norm for $q>1$, contrativity in $L^{1}$, global existence, decay to zero in various norms when $t \rightarrow \infty$, in case of global existence, etc, even in the case of regularized equation. In fact, problem (1) could be much more complicated when $f$ depends on $x$ and, $-f^{\prime}(x) \gg 1$, as illustrated in [6] for the one-dimensional case.

The results presented in this work are original and extend previous that are already in the literature, see, as exemple [7], where the autor proof the analogous results only for non-negative solutions and for the one-dimensional case. Besides that, in contrast to the current literature (see e.g. $[8,9,10]$ ), this kind of interaction (with mass conservation or similar links) only began to be mathematically investigated very recently (in $[1,2,7])$.

This paper is organized as follows: Section 2 is devoted to showing that $L^{1}$ norm of the smooth bounded solutions decreases on time, already in the Section 3, we will show that these solutions have mass conservation property. In both Sections, we will consider $u_{0}>0$ (or $u_{0}<0$ ) for all $x \in \mathbb{R}^{n}$. Finally, Section 4 is devoted to extend these results to signed solutions.

We remark that, in this paper, we understand as smooth and weak solution to the problem (1) a function that satisfies the following definitions, respectively: 
Definition 1.1. A smooth function $u \in L_{l o c}^{\infty}\left(\left[0, T_{*}\right), L^{\infty}\left(\mathbb{R}^{n}\right)\right)$ is a bounded classical solution in a maximal interval of existence $\left[0, T_{*}\right)$, where $0 \leq T_{*} \leq \infty$, if it satisfies classically the first equation of (1) and, besides that, $u(\cdot, t) \rightarrow u_{0}$ in $L_{l o c}^{1}\left(\mathbb{R}^{n}\right)$, when $t \rightarrow 0$.

Definition 1.2. $A$ weak solution to the problem (1) is a function $u$ that satisfies

$$
\int_{0}^{T} \int_{\mathbb{R}^{n}} u(x, \tau) \Psi_{t}(x, \tau)+\langle f(x, \tau, u), \nabla \Psi(x, \tau)\rangle+\frac{|u(x, \tau)|^{\alpha}}{\alpha+1} u(x, \tau) \Delta \Psi(x, \tau) d x d \tau=0,
$$

for any $\Psi \in C_{0}^{\infty}\left(\mathbb{R}^{n} \times(0, T)\right)$ and $u(\cdot, t) \rightarrow u_{0}$ in $L_{l o c}^{1}$, when $t \rightarrow 0$.

When $u_{0}>0$ (or $u_{0}<0$ ) for all $x \in \mathbb{R}^{n}$, the solutions of the problem (1) are strictly positive (or strictly negative), see [7]. In these cases the solutions are smooth, which is the reason why we consider smooth solutions in Sections 2 and 3, and weak solutions in Section 4 where the initial data and solutions can change sign. For a more complete discussion of regularity see e.g. $[3,4,5,11,13]$.

\section{Decreasing $L^{1}$ norm}

In this section, we show that the $L^{1}\left(\mathbb{R}^{n}\right)$ norm of a smooth solution of (1) decreases while it exists, that is, $\|u(\cdot, t)\|_{L^{1}\left(\mathbb{R}^{n}\right)} \leq\left\|u_{0}\right\|_{L^{1}\left(\mathbb{R}^{n}\right)}, \forall 0<t<T_{*}$, where $\left[0, T_{*}\right]$ is the maximal interval of existence.

(f1) Let be $M>0$, and $T<T_{*}$. Then, for all $(x, t, v) \in \mathbb{R}^{n} \times[0, T] \times[-M, M]$, exists $K=K(M, T)>0$ such that $|f(x, t, v)|<K|v|$.

Theorem 2.1. Let $u(x, t) \in L_{l o c}^{\infty}\left(\left[0, T_{*}\right], L^{\infty}\left(\mathbb{R}^{n}\right)\right)$ be a smooth solution of (1) $t$. If $f$ satisfies (f1), then

$$
\|u(\cdot, t)\|_{L^{1}\left(\mathbb{R}^{n}\right)} \leq\left\|u_{0}\right\|_{L^{1}\left(\mathbb{R}^{n}\right)}, \forall 0<t<T_{*} .
$$

Proof. Let $S \in C^{1}(\mathbb{R})$ be an odd increasing function, such that $S(\mathrm{u})=-1$ if $\mathrm{u} \leq-1, S(\mathrm{u})=1$ if $\mathrm{u} \geq 1$ and $-1 \leq S \leq 1$. Let be $\delta>0$, we define a function $L_{\delta} \in C^{2}(\mathbb{R})$ by

$$
L_{\delta}(\mathrm{u}):=\int_{0}^{\mathrm{u}} S(\mathrm{v} / \delta) d \mathrm{v}, \quad \mathrm{u} \in \mathbb{R} .
$$

Let be $R>0$ and $\varepsilon>0$. We define a function $\zeta_{R, \varepsilon}: \mathbb{R}^{n} \rightarrow \mathbb{R}$ by

$$
\zeta_{R, \varepsilon}(x)= \begin{cases}\exp \left(-\varepsilon \sqrt{1+|x|^{2}}\right)-\exp \left(-\varepsilon \sqrt{1+R^{2}}\right), & \text { if }|x|<R, \\ 0, & \text { if }|x| \geq R .\end{cases}
$$

Let $u(x, t)$ be a smooth solution of the problem (1) and let be $t_{0} \in(0, T]$ such that $T \in\left(t_{0}, T_{*}\right)$. Multiplying the first equation of (1) by $\zeta_{R}(x) L_{\delta}^{\prime}(u)$ (for simplicity, we will denote $\zeta_{R, \varepsilon}$ by $\zeta_{R}$ ) and integrating 
on $\mathbb{R}^{n} \times\left[t_{0}, t\right]$, we get

$$
\begin{aligned}
\int_{t_{0}}^{t} \int_{|x| \leq R} L_{\delta}^{\prime}(u) u_{t} \zeta_{R}(x) d x d \tau+\int_{t_{0}}^{t} \int_{|x| \leq R} L_{\delta}^{\prime}(u) \operatorname{div}(f(x, \tau, u)) \zeta_{R}(x) d x d \tau & \\
& =\int_{t_{0}}^{t} \int_{|x| \leq R} L_{\delta}^{\prime}(u) \operatorname{div}\left(|u|^{\alpha} \nabla u\right) \zeta_{R}(x) d x d \tau
\end{aligned}
$$

because $\operatorname{supp} \zeta_{R} \subset B_{R}:=\left\{x \in \mathbb{R}^{n}:|x|<R\right\}$.

As $\zeta_{R}(x)=0$ for $|x|=R$, using Fubini's Theorem and the Divergence Theorem, we have

$$
\begin{aligned}
\int_{|x| \leq R} L_{\delta}(u) \zeta_{R}(x) d x= & \int_{|x| \leq R} L_{\delta}\left(u\left(x, t_{0}\right)\right) \zeta_{R}(x) d x \\
& +\int_{t_{0}}^{t} \int_{|x| \leq R} L_{\delta}^{\prime \prime}(u) \zeta_{R}(x)\langle\nabla u, f(x, \tau, u)\rangle d x d \tau \\
& +\int_{t_{0}}^{t} \int_{|x| \leq R} L_{\delta}^{\prime}(u)\left\langle\nabla \zeta_{R}(x), f(x, \tau, u)\right\rangle d x d \tau \\
& -\int_{t_{0}}^{t} \int_{|x| \leq R} L_{\delta}^{\prime \prime}(u)\langle\nabla u, \nabla u\rangle|u|^{\alpha} \zeta_{R}(x) d x d \tau \\
& -\int_{t_{0}}^{t} \int_{|x| \leq R} L_{\delta}^{\prime}(u)|u|^{\alpha}\left\langle\nabla \zeta_{R}(x), \nabla u\right\rangle d x d \tau
\end{aligned}
$$

As $-\int_{t_{0}}^{t} \int_{|x| \leq R} L_{\delta}^{\prime \prime}(u)\langle\nabla u, \nabla u\rangle|u|^{\alpha} \zeta_{R}(x) d x d \tau \leq 0$, we obtain

$$
\begin{aligned}
\int_{|x| \leq R} L_{\delta}(u) \zeta_{R}(x) d x \leq & \int_{|x| \leq R} L_{\delta}\left(u\left(x, t_{0}\right)\right) \zeta_{R}(x) d x \\
& +\int_{t_{0}}^{t} \int_{|x| \leq R} L_{\delta}^{\prime \prime}(u) \zeta_{R}(x)\langle\nabla u, f(x, \tau, u)\rangle d x d \tau \\
& +\int_{t_{0}}^{t} \int_{|x| \leq R} L_{\delta}^{\prime}(u)\left\langle\nabla \zeta_{R}(x), f(x, \tau, u)\right\rangle d x d \tau \\
& -\int_{t_{0}}^{t} \int_{|x| \leq R} L_{\delta}^{\prime}(u)|u|^{\alpha}\left\langle\nabla \zeta_{R}(x), \nabla u\right\rangle d x d \tau .
\end{aligned}
$$

Let be

$$
\begin{aligned}
& I_{1}(t)=\int_{t_{0}}^{t} \int_{|x| \leq R} L_{\delta}^{\prime \prime}(u) \zeta_{R}(x)\langle\nabla u, f(x, \tau, u)\rangle d x d \tau, \\
& I_{2}(t)=\int_{t_{0}}^{t} \int_{|x| \leq R} L_{\delta}^{\prime}(u)\left\langle\nabla \zeta_{R}(x), f(x, \tau, u)\right\rangle d x d \tau \text { and } \\
& I_{3}(t)=-\int_{t_{0}}^{t} \int_{|x| \leq R} L_{\delta}^{\prime}(u)|u|^{\alpha}\left\langle\nabla \zeta_{R}(x), \nabla u\right\rangle d x d \tau .
\end{aligned}
$$

Now, we will obtain estimates for each integrals $I_{i}(t)$, for $i=1,2,3$, defined above. We will start with $I_{1}(t)$. 
As $u(x, t) \in L_{l o c}^{\infty}\left(\left[0, T_{*}\right], L^{\infty}\left(\mathbb{R}^{n}\right)\right)$, and $u$ is smooth $\exists M=M(T)>0$ such that $|u(x, t)| \leq M, \forall x \in \mathbb{R}^{n}$ and $\forall t \in[0, T]$. By hypotheses (f1) and applying Cauchy-Schwarz's inequality, we have

$I_{1}(t)=\int_{t_{0}}^{t} \int_{|x| \leq R} L_{\delta}^{\prime \prime}(u) \zeta_{R}(x)\langle\nabla u, f(x, \tau, u)\rangle d x d \tau \leq K(M, T) \int_{t_{0}}^{t} \int_{|x| \leq R}\left|L_{\delta}^{\prime \prime}(u) u\right| \zeta_{R}(x)|\nabla u| d x d \tau$.

Observe that $\left|L_{\delta}^{\prime \prime}(v) v\right| \leq C$, and $\lim _{\delta \rightarrow 0} L_{\delta}^{\prime \prime}(v) v=0$ uniformly in $v \in \mathbb{R}$. Since $u$ is smooth, $|\nabla u(x, \tau)| \leq C_{1}, \forall x \in B_{R}$ and $\forall \tau \in\left[t_{0}, t\right]$.

So, by Lebesgue's Dominated Convergence Theorem, we have

$$
I_{1}(t)=\int_{t_{0}}^{t} \int_{|x| \leq R} L_{\delta}^{\prime \prime}(u) \zeta_{R}(x)\langle\nabla u, f(x, \tau, u)\rangle d x d \tau \rightarrow 0, \text { when } \delta \rightarrow 0 .
$$

For the second term, we get

$\left|I_{2}(t)\right|=\left|\int_{t_{0}}^{t} \int_{|x| \leq R} L_{\delta}^{\prime}(u)\left\langle\nabla \zeta_{R}(x), f(x, \tau, u)\right\rangle d x d \tau\right| \leq K(M, T) \int_{t_{0}}^{t} \int_{|x| \leq R}\left|L_{\delta}^{\prime}(u) u \nabla \zeta_{R}(x)\right| d x d \tau$.

Since $\left|L_{\delta}^{\prime}(u)\right| \leq 1$ and $\left|\nabla \zeta_{R}\right| \leq \varepsilon e^{-\varepsilon \sqrt{1+|x|^{2}}}$, we have

$$
\left|I_{2}(t)\right| \leq \varepsilon K(M, T) \int_{t_{0}}^{t} \int_{|x| \leq R}|u(x, \tau)| e^{-\varepsilon \sqrt{1+|x|^{2}}} d x d \tau .
$$

Now, we will obtain an estimate for the third term. Since $\lim _{\delta \rightarrow 0} L_{\delta}^{\prime}(u)=\operatorname{sgn}(u)$, letting $\delta \rightarrow 0$, we get, by Lebesgue's Dominated Convergence Theorem,

$I_{3}(t)=-\int_{t_{0}}^{t} \int_{|x| \leq R} L_{\delta}^{\prime}(u)|u|^{\alpha}\left\langle\nabla \zeta_{R}(x), \nabla u\right\rangle d x d \tau \longrightarrow-\int_{t_{0}}^{t} \int_{|x| \leq R} \operatorname{sgn}(u)|u|^{\alpha}\left\langle\nabla \zeta_{R}(x), \nabla u\right\rangle d x d \tau$.

Since $\nabla\left(|u|^{\beta+1}\right)=(\beta+1)|u|^{\beta} \operatorname{sgn}(u) \nabla u, \forall \beta>0$, we have

$I_{3}(t)=-\int_{t_{0}}^{t} \int_{|x| \leq R} \operatorname{sgn}(u)|u|^{\alpha}\left\langle\nabla \zeta_{R}(x), \nabla u\right\rangle d x d \tau=\frac{-1}{\alpha+1} \int_{t_{0}}^{t} \int_{|x| \leq R}\left\langle\nabla \zeta_{R}(x), \nabla\left(|u|^{\alpha+1}\right)\right\rangle d x d \tau$.

Applying the Divergence Theorem, we obtain 
$I_{3}(t)=\frac{1}{\alpha+1} \int_{t_{0}}^{t} \int_{|x| \leq R} \Delta \zeta_{R}(x)|u|^{\alpha+1} d x d \tau-\frac{1}{\alpha+1} \int_{t_{0}}^{t} \int_{|x|=R}|u|^{\alpha+1}\left\langle\nabla \zeta_{R}(x), \frac{x}{R}\right\rangle d \sigma(x) d \tau$

Since $\left|\Delta \zeta_{R}(x)\right| \leq 2 n \varepsilon e^{-\varepsilon \sqrt{1+|x|^{2}}}$ and $\left|\nabla \zeta_{R}(x)\right| \leq \varepsilon e^{-\varepsilon \sqrt{1+|x|^{2}}}$, we have

$$
\left|I_{3}(t)\right| \leq \frac{2 n \varepsilon M^{\alpha+1}(T)}{\alpha+1} \int_{t_{0}}^{t} \int_{|x| \leq R}|u(x, \tau)| e^{-\varepsilon \sqrt{1+|x|^{2}}} d x d \tau+\frac{M^{\alpha+1}(T)}{\alpha+1}\left(t-t_{0}\right) \varepsilon R^{n-1} n \omega_{n} e^{-\varepsilon \sqrt{1+R^{2}}},
$$

where $\omega_{n}$ is the volume of the unit ball in $\mathbb{R}^{n}$. Note that

$$
\frac{M^{\alpha+1}(T)}{\alpha+1}\left(t-t_{0}\right) \varepsilon R^{n-1} n \omega_{n} e^{-\varepsilon \sqrt{1+R^{2}}} \rightarrow 0, \text { when } R \rightarrow \infty .
$$

Since $\int_{|x| \leq R} L_{\delta}(u) \zeta_{R}(x) d x=\int_{|x| \leq R} L_{\delta}\left(u\left(x, t_{0}\right)\right) \zeta_{R}(x) d x+I_{1}(t)+I_{2}(t)+I_{3}(t)$, applying the triangle inequality, we get

$$
\int_{|x| \leq R} L_{\delta}(u) \zeta_{R}(x) d x \leq \int_{|x| \leq R} L_{\delta}\left(u\left(x, t_{0}\right)\right) \zeta_{R}(x) d x+\left|I_{1}(t)\right|+\left|I_{2}(t)\right|+\left|I_{3}(t)\right| .
$$

Letting $\delta \rightarrow 0$ and using (5), (6) and (7), and letting $t_{0} \rightarrow 0$, in this order, we obtain

$$
\begin{aligned}
\int_{|x| \leq R}|u(x, t)| \zeta_{R}(x) d x \leq \int_{|x| \leq R}\left|u_{0}(x)\right| \zeta_{R}(x) d x & +\varepsilon \int_{0}^{t} S(T) \int_{|x| \leq R}|u(x, \tau)| e^{-\varepsilon \sqrt{1+|x|^{2}}} d x d \tau \\
& +\varepsilon\left(t-t_{0}\right) R^{n-1} n \omega_{n} e^{-\varepsilon \sqrt{1+R^{2}}}\left(\frac{M^{\alpha+1}(T)}{\alpha+1}\right),
\end{aligned}
$$

where $S(T)=K(M, T)+\frac{2 n M^{\alpha}(T)}{\alpha+1}$.

Letting $R \rightarrow \infty$, we obtain

$$
\int_{\mathbb{R}^{n}}|u(x, t)| \Psi_{\varepsilon}(x) d x \leq \int_{\mathbb{R}^{n}}\left|u_{0}(x)\right| \Psi_{\varepsilon}(x) d x+\varepsilon S(T) \int_{0}^{t} \int_{\mathbb{R}^{n}}|u(x, \tau)| \Psi_{\varepsilon}(x) d x d \tau,
$$

where $\Psi_{\varepsilon}(x)=e^{-\varepsilon \sqrt{1+|x|^{2}}}$.

\section{By Gronwall's Theorem, we have}

$$
\int_{\mathbb{R}^{n}}|u(x, t)| \Psi_{\varepsilon}(x) d x \leq \exp (\varepsilon S(T) T) \int_{\mathbb{R}^{n}}\left|u_{0}(x)\right| \Psi_{\varepsilon}(x) d x .
$$

Letting $\varepsilon \rightarrow 0$, we get $\int_{\mathbb{R}^{n}}|u(x, t)| d x \leq \int_{\mathbb{R}^{n}}\left|u_{0}(x)\right| d x$. Then,

$$
\|u(\cdot, t)\|_{L^{1}\left(\mathbb{R}^{n}\right)} \leq\left\|u_{0}\right\|_{L^{1}\left(\mathbb{R}^{n}\right)}, \quad \forall t \in[0, T]
$$




\section{Mass conservation}

In this section, we will proof mass conservation property for bounded smooth solutions of (1), that is, if $u(x, t)$ is a bounded smooth solution of (1), then $\int_{\mathbb{R}^{n}} u(x, t) d x=\int_{\mathbb{R}^{n}} u_{0}(x) d x$.

Theorem 3.1. Let be $u$ a bounded smooth solution of (1). If $f$ satisfies (f1) then

$$
\int_{\mathbb{R}^{n}} u(x, t) d x=\int_{\mathbb{R}^{n}} u_{0}(x) d x .
$$

Proof. Let be $\psi \in C^{\infty}(\mathbb{R})$ such that $\psi(y)=0, \forall y \leq 0$ and $\psi(y)=1, \forall y \geq 1$ with $0 \leq \psi \leq 1$, $\max _{x \in \mathbb{R}^{n}}\left|\psi^{\prime}(x)\right|=M_{1}<\infty$ and $\max _{x \in \mathbb{R}^{n}}\left|\psi^{\prime \prime}(x)\right|=M_{2}<\infty$. Let be $R>0$, we define

$$
\bar{\zeta}_{R}(x)= \begin{cases}1, & \text { if }|x|<R \\ 0, & \text { if }|x|>2 R \\ \psi\left(\frac{|x|}{R}-1\right) & \text { if } R \leq|x| \leq 2 R\end{cases}
$$

Let be $u$ a bounded smooth solution of (1) with $|u| \leq M$ and $t_{0} \in(0, T]$ such that $T \in\left(t_{0}, T_{*}\right)$. Multiplying the first equation of (1) by $\bar{\zeta}_{R}(x) \in C^{\infty}\left(\mathbb{R}^{n}\right)$ and integrating in $\mathbb{R}^{n} \times\left[t_{0}, t\right]$, we have

$$
\int_{t_{0}}^{t} \int_{|x| \leq 2 R} u_{t} \bar{\zeta}_{R}(x) d x d \tau+\int_{t_{0}}^{t} \int_{|x| \leq 2 R} \operatorname{div}(f(x, \tau, u)) \bar{\zeta}_{R}(x) d x d \tau=\int_{t_{0}}^{t} \int_{|x| \leq 2 R} \operatorname{div}\left(|u|^{\alpha} \nabla u\right) \bar{\zeta}_{R}(x) d x d \tau
$$

Since $\bar{\zeta}_{R}(x)=0$ for $|x|>2 R$, using Fubini's Theorem and the Divergence Theorem, we get

$$
\begin{array}{r}
\int_{|x| \leq 2 R} u(x, t) \bar{\zeta}_{R}(x) d x=\int_{|x| \leq 2 R} u\left(x, t_{0}\right) \bar{\zeta}_{R}(x) \\
d x+\int_{t_{0}}^{t} \int_{|x| \leq 2 R}\left\langle\nabla \bar{\zeta}_{R}(x), f(x, \tau, u)\right\rangle d x d \tau \\
-\int_{t_{0}}^{t} \int_{|x| \leq 2 R}|u(x, t)|^{\alpha}\left\langle\nabla u, \nabla \bar{\zeta}_{R}(x)\right\rangle d x d \tau .
\end{array}
$$

Let be

$$
\begin{aligned}
& I_{1}(t)=\int_{t_{0}}^{t} \int_{|x| \leq 2 R}\left\langle\nabla \bar{\zeta}_{R}(x), f(x, \tau, u)\right\rangle d x d \tau \text { and } \\
& I_{2}(t)=-\int_{t_{0}}^{t} \int_{|x| \leq 2 R}|u(x, t)|^{\alpha}\left\langle\nabla u, \nabla \bar{\zeta}_{R}(x)\right\rangle d x d \tau .
\end{aligned}
$$

Now, we will show that $\left|I_{1}\right| \rightarrow 0$ and $\left|I_{2}\right| \rightarrow 0$, when $R \rightarrow \infty$. We will start with $I_{1}$. Using the Cauchy-Schwarz Inequality and (f1), we obtain

$$
\left|I_{1}(t)\right| \leq \frac{K(M, T) M_{1}}{R} \int_{t_{0}}^{t} \int_{\mathbb{R}^{n}}|u(x, \tau)| d x d \tau,
$$


as $\left|\nabla \bar{\zeta}_{R}(x)\right| \leq \frac{M_{1}}{R}$ and $|f(x, \tau, u)|<K(M, T)|u(x, t)|$. By Theorem 2.1, we have $\int_{\mathbb{R}^{n}}|u(\cdot, t)| d x \leq \int_{\mathbb{R}^{n}}\left|u_{0}(x)\right| d x$. Then, letting $R \rightarrow \infty$, we have $I_{1}(t) \rightarrow 0$.

Now, we will estimate $I_{2}(t)$, to this end, we define $J(x, t)=\int_{0}^{u(x, t)}|v|^{\alpha} d v$, and so $\nabla J(x, t)=|u(x, t)|^{\alpha} \nabla u$. Then

$I_{2}(t)=-\int_{t_{0}}^{t} \int_{|x| \leq 2 R}|u(x, t)|^{\alpha}\left\langle\nabla u, \nabla \bar{\zeta}_{R}(x)\right\rangle d x d \tau=-\int_{t_{0}}^{t} \int_{|x| \leq 2 R}\left\langle\nabla J(x, t), \nabla \bar{\zeta}_{R}(x)\right\rangle d x d \tau$.

Appling The Divergence Theorem, we obtain

$$
I_{2}(t)=\int_{t_{0}}^{t} \int_{|x| \leq 2 R} J(x, t) \Delta \bar{\zeta}_{R}(x) d x d \tau-\int_{t_{0}}^{t} \int_{|x|=2 R} J(x, t)\left\langle\nabla \bar{\zeta}_{R}(x), \frac{x}{2 R}\right\rangle d \sigma(x) d \tau
$$

Since $\left|\Delta \bar{\zeta}_{R}(x)\right| \leq \frac{M_{2}}{R^{2}}$ and $|J(x, t)| \leq \frac{1}{\alpha+1}|u(x, t)|^{\alpha+1}$, we get

$$
\begin{aligned}
\left|I_{2}(t)\right| & \leq \frac{M_{2}}{R^{2}(\alpha+1)} \int_{t_{0}}^{t} \int_{\mathbb{R}^{n}}|u(x, \tau)|^{\alpha+1} d x d \tau+\frac{M_{1}}{R(\alpha+1)} \int_{t_{0}}^{t} \int_{|x|=2 R}|u(x, \tau)|^{\alpha+1} d \sigma(x) d \tau \\
& \leq \frac{M_{2}}{R^{2}(\alpha+1)}\left\|u_{0}\right\|_{L^{1}\left(\mathbb{R}^{n}\right)}^{\alpha+1}\left(t-t_{0}\right)+\frac{M_{1}}{R(\alpha+1)}\left\|u_{0}\right\|_{L^{1}\left(\mathbb{R}^{n}\right)}^{\alpha+1}\left(t-t_{0}\right) .
\end{aligned}
$$

Then, letting $R \rightarrow \infty$ we have $\left|I_{2}(t)\right| \rightarrow 0$.

Therefore, letting $R \rightarrow \infty$, we get from (8) that

$$
\int_{\mathbb{R}^{n}} u(x, t) d x=\int_{\mathbb{R}^{n}} u\left(x, t_{0}\right) d x
$$

since $I_{1}(t)$ and $I_{2}(t)$ go to zero, when $R \rightarrow \infty$, and $\bar{\zeta}_{R}(x) \rightarrow 1$, when $R \rightarrow \infty$.

\section{Decreasing $L^{1}$ norm for signed solutions}

In this section, we will obtain the decrease of the norm $L^{1}$ and the conservation of mass for weak signed solutions of the problem

$$
\begin{aligned}
u_{t}+\operatorname{div} f(x, t, u) & =\operatorname{div}\left(|u(x, t)|^{\alpha} \nabla u\right) \quad x \in \mathbb{R}^{n}, t>0, \\
u(\cdot, 0) & =u_{0} \in L^{1}\left(\mathbb{R}^{n}\right) \cap L^{\infty}\left(\mathbb{R}^{n}\right) .
\end{aligned}
$$


where $u_{0}$ is any function in $L^{1}(\mathbb{R})$.

Let us consider the auxiliary problems

$$
\begin{gathered}
u_{t}+\operatorname{div} f(x, t, u)=\operatorname{div}\left(|u(x, t)|^{\alpha} \nabla u\right) \quad x \in \mathbb{R}^{n}, t>0, \\
u(\cdot, 0)=u_{0}^{+}+\epsilon \psi \in L^{1}\left(\mathbb{R}^{n}\right) \cap L^{\infty}\left(\mathbb{R}^{n}\right) .
\end{gathered}
$$

and

$$
\begin{aligned}
u_{t}+\operatorname{div} f(x, t, u) & =\operatorname{div}\left(|u(x, t)|^{\alpha} \nabla u\right) \quad x \in \mathbb{R}^{n}, t>0, \\
u(\cdot, 0) & =-u_{0}^{-}-\epsilon \psi \in L^{1}\left(\mathbb{R}^{n}\right) \cap L^{\infty}\left(\mathbb{R}^{n}\right) .
\end{aligned}
$$

where $\epsilon>0$ and $0<\psi \in L^{1}\left(\mathbb{R}^{n}\right) \cap L^{\infty}\left(\mathbb{R}^{n}\right)$.

To prove the main result of this article, we need $f$ to satisfy the hypotheses below

$$
|f(x, t, u)-f(x, t, v)| \leq C_{f}(M, T)|u-v|, \quad \forall x \in \mathbb{R}^{n}, 0 \leq t \leq T .
$$

Theorem 4.1. Let be $T>0$. If $u(x, t)$ is a weak and bounded solution in $\mathbb{R}^{n} \times[0, T]$ of (9) and $f$ satisfies (f1 - f2), then

$$
\|u(\cdot, t)\|_{L^{1}\left(\mathbb{R}^{n}\right)} \leq\left\|u_{0}\right\|_{L^{1}\left(\mathbb{R}^{n}\right)}, \forall t \in(0, T]
$$

and

$$
\int_{\mathbb{R}^{n}} u_{0}^{-}(x) d x \leq \int_{\mathbb{R}^{n}} u(x, t) d x \leq \int_{\mathbb{R}^{n}} u_{0}^{+}(x) d x .
$$

Proof. Let $u, v, w$ be, respectively, solutions of (9), (10) and (11), by comparison (see [7]), we have $w(x, t) \leq u(x, t) \leq v(x, t), w(x, t) \leq 0$ and $0 \leq v(x, t), \forall x \in \mathbb{R}^{n}$ and $\forall t \in[0, T]$. So, $|u(x, t)| \leq|w(x, t)-v(x, t)|$. Now, by Theorem 2.1, the estimates obtained for smooth solutions, that do not change sign, of problems with initial data that do not change sign, it follows that

$$
\begin{gathered}
\int_{\mathbb{R}^{n}}|u(x, t)| d x \leq \int_{\mathbb{R}^{n}}|v(x, t)-w(x, t)| d x \leq \int_{\mathbb{R}^{n}}\left|u_{0}^{+}(x)+u_{0}^{-}(x)+2 \epsilon \psi(x)\right| d x, \forall t \in(0, T] . \\
\text { As }\left\|u_{0}^{+}+u_{0}^{-}+2 \epsilon \psi\right\|_{L^{1}\left(\mathbb{R}^{n}\right)} \rightarrow\left\|u_{0}\right\|_{L^{1}\left(\mathbb{R}^{n}\right)} \text {, when } \epsilon \rightarrow 0^{+}, \text {we obtain } \\
\|u(\cdot, t)\|_{L^{1}\left(\mathbb{R}^{n}\right)} \leq\left\|u_{0}\right\|_{L^{1}\left(\mathbb{R}^{n}\right)}, \forall t \in(0, T] .
\end{gathered}
$$

The second statement immediately follows from $w(x, t) \leq(x, t) \leq v(x, t)$, it is just integrate in $R^{n}$ and letting epsilon go to zero. 


\section{Conclusions}

Note that we do not prove the mass conservation for signed solutions, we leave this question open. We believe that signed solutions has mass conservation.

The $L^{1}$ norm decreasing in addition with others properties can provide us qualitative information about signed solutions, for example, if we have the smoothing effect $\|u(\cdot, t)\|_{L^{\infty}} \leq F(x, t, u)\|u(\cdot, t)\|_{L^{1}}$, where $F(x, t, u) \leq K$ in every set $\mathbb{R}^{n} \times\left[0, T_{*}\right] \times[-M, M]$, then every weak (signed) solution is bounded, in addition, the solution is bounded globaly in case global existence.

Other contribuition of this paper is in the study of stacionary solutions, every stacionary solution, if it exists, has mass between $-\int u_{0}^{-} d x$ and $\int u_{0}^{+} d x$.

\section{Bibliography}

[1] Barrionuevo, J. A.; Oliveira, L. S.; Zingano, P. R. General asymptotic supnorm estimates for solutions of one-dimensional advection-diffusion equations in heterogeneous media.

International Journal of Partial Differential Equations, v. 2014, 8 p., DOI:

http://dx.doi.org/10.1155/2014/450417.

[2] Braz e Silva, P.; Melo, W.; Zingano, P. R. An asymptotic supnorm estimate for solutions of 1-D systems of convection-diffusion equations. Journal of Differential Equations, v. 258, n. 8, p. 2806-2822, 2015. DOI: 10.1016/j.jde.2014.12.026.

[3] Daskalopoulos, P.; KeniG, C. E.Degenerate Diffusions: initial value problems and local regularity theory. Zürich: European Mathematical Society, 2007.

[4] DiBenedetto, E. Degenerate Parabolic Equations. New York: Springer, 1993.

[5] DiBenedetto, E. On the local behavior of solutions of degenerate parabolic equations with measurable coefficients. Annali della Scuola Normale Superiore di Pisa, v. 13, p. 487-535, 1986.

[6] DIEHL, N. M. L. Contributions to the theory of equations of porous media with advective terms(in Portuguese). PhD Thesis - Programa de Pós-Graduação em Matemática, Universidade Federal do Rio Grande do Sul, Porto Alegre, RS, Brazil, September/2015.

[7] FABRIS, L. On the global existence and supnorm estimates for nonnegative solutions of the porous medium equation with arbitrary advection terms (in Portuguese). PhD Thesis Programa de Pós-Graduação em Matemática, Universidade Federal do Rio Grande do Sul, Porto Alegre, RS, Brazil, October/2013.

[8] HU, B. Blow-up Theories for Semilinear Parabolic Equations. Berlin: Springer, 2011. 
[9] Quittner, P.; Souplet, P. Superlinear Parabolic Problems: blow-up, global existence and steady states. Basel: Birkhäuser, 2007.

[10] Samarskil, A. A.; Galaktionov, V. A.; Kurdyumov, S.; Mikhailov, A. P. Blow-up in Quasilinear Parabolic Equations. Berlin: Walter de Gruyter, 1995.

[11] URBANO, J. M. The Method of Intrinsic Scaling: a systematic approach to regularity for degenerate and singular PDEs. Lecture Notes in Mathematics. v. 1930. New York: Springer, 2008.

[12] VÁZqUEZ, J. L. Smoothing and Decay Estimates for Nonlinear Diffusion Equations: Equations of Porous Medium Type. Oxford: Oxford University Press, 2006.

[13] VÁzQUEZ, J. L. The Porous Medium Equation: mathematical theory. Oxford: Oxford University Press, 2007. 